\title{
The Effect of Diabetes Mellitus, Insulin, and Thiazolidinediones on Bone Histomorphometry in Streptozotocin-induced Diabetic Postmenopausal Wistar Rats
}

Corresponding Author:

Derya Köseoğlu;

Buharavler Mah Buhara 5.

Cad No:34/12 Çorum/Turkey

Tel: $+90-5056104214$

email:

drderyaksgl@gmail.com

Received 7 January 2021

Accepted 10 March 2021

Published 31 March 2021

Production and Hosting by

Knowledge E

(c) Derya Köseoğlu et al. This article is distributed under the terms of the Creative

Commons Attribution

which permits

unrestricted use and

redistribution provided that

the original author and

source are credited.

Editor-in-Chief:

Prof. Mohammad A. M. Ibnouf

\section{Derya Köseoğlư ${ }^{1}$, Gülnur Take Kaplanoğlu ${ }^{2}$, Banu Aktaș Yılmaz ${ }^{3}$, Erdal Kan ${ }^{4}$, and Nuri Çakır ${ }^{5,4}$}

${ }^{1}$ Department of Endocrinology and Metabolism, Faculty of Medicine, Hitit University, Çorum, Turkey

${ }^{2}$ Department of Histology, Faculty of Medicine, Gazi University, Yenimahalle/Ankara, Turkey

${ }^{3}$ Department of Endocrinology and Metabolism, Faculty of Medicine, Ufuk University, Gölbașı/Ankara, Turkey

${ }^{4}$ Department of Endocrinology and Metabolism, Medicana Samsun Hospital, Canik/Samsun, Turkey

${ }^{5}$ Department of Endocrinology and Metabolism, Faculty of Medicine, Gazi University, Yenimahalle/Ankara, Turkey

ORCID:

Derya Köseoğlu: http://orcid.org/0000-0002-1080-3208

\section{Abstract}

Background: Osteoporosis is a metabolic skeletal disease with low bone mass and bone microarchitectural disorganization. Thiazolidinediones (TZD) increase insulin sensitivity through activation of peroxisome proliferator-activated receptor gamma (PPARY). One of the most important side effects of this drugs is its effects on bone, especially in postmenopausal women. The purpose of this study was to evaluate the effect of diabetes mellitus (DM), insulin, and TZDs on bone in postmenopausal Wistar rats.

Methods: Sixteen postmenopausal Wistar rats were divided into four groups: (i) control group, (ii) Streptozotocin-induced DM group without treatment, (iii) Streptozotocininduced DM group with insulin therapy, and (iv) Streptozotocin-induced DM group receiving rosiglitazone. Pictures of the obtained samples were taken under computerequipped photo-light microscope, and bone tissue ratios were calculated in an area of $1 \mathrm{~mm}^{2}$. In this area, trabecular thicknesses were measured from six randomly selected regions. In addition, femoral neck regions were determined by measuring the farthest distance.

Results: Compared to the control group, trabecular thicknesses were decreased in the uncontrolled DM and rosiglitazone groups. In the rosiglitazone-treated group, trabecular thickness was decreased compared to the uncontrolled DM group. The histological examination of the bones showed that uncontrolled DM and rosiglitazone treatment negatively affected the osteoblast and osteocyte activity. Insulin-treated group had a similar histologic examination compared to the control group.

Conclusion: Our study showed that DM had unfavorable effects on bones, and rosiglitazone further exerts this effect. However, the negative effect of DM may be neutralized with the use of insulin. 


\section{Introduction}

It is well-known that there is an increase in bone fragility in patients with type-1 diabetes mellitus (DM) [1, 2]. However, there is no data about type-2 DM and bone as much as type$1 \mathrm{DM}$, and the assumption that obesity frequently accompanying in type-2 DM patients protects patients against bone fracture was accepted [3, 4]. However, epidemiological studies have shown that, despite the normal-high bone mineral density (BMD), the risk of fractures increases in type-2 DM patients. These studies show an increased risk of fracture in non-vertebral regions, especially hip, proximal humerus, and foot $[1,3,5,6]$.

Thiazolidinedione (TZD) is an oral antidiabetic drug group that increases insulin sensitivity by activation of peroxisome proliferator-activating receptor gamma (PPARY). PPARY is a group of receptors that have important effects on bone metabolism $[7,8]$. In vitro studies have shown that PPARY agonists increase adipogenesis by reducing osteoblastogenesis over the PPARY2 receptor. Gimble et al. showed that expression of adipocyte-specific genes and adipocyte count increased with the addition of pioglitazone and rosiglitazone to the cell medium containing bone marrow stromal cells [7]. It was found that there were fewer adipocytes and more osteoblasts in the bone marrow stromal cell cultures containing PPARY insensitive cells $[9,10]$. In studies carried out with osteoclasts, PPARY agonists have been shown to reduce osteoclast formation [10, 11]. Similar relationships were found in animal studies. With the use of TZD, increased bone marrow adipocyte count and proportionally decreased bone formation and increased bone resorption were detected [12-15].

In literature, studies showing the effects of DM and TZD therapies for blood glucose $(B G)$ regulation on bone have been reported $[5,6,8,12,13]$. Postmenopausal animals were selected for the purpose of this study since the negative effects of TZDs (rosiglitazone and pioglitazone) on bone are seen more prominently in cases of estrogen deficiency [15]. This study was aimed at clarifying the relationship between DM and the drugs used in the treatment for DM with bone fragility through detailed examinations (examination of histomorphometric parameters with light microscope, histological evaluation) of the effects of DM, BG regulation with insulin, and TZDs on the bone. The effect of DM, insulin, and rosiglitazone on bone histomorphometric parameters in postmenopausal streptozotocin-induced diabetic Wistar rats have not formerly been investigated in the literature. 


\section{Materials and Methods}

This study was performed at the Faculty of Medicine Experimental Animals Research Laboratory of Gazi University.

A total of 16 albino Wistar postmenopausal rats were included in the study. All animals were kept in polycarbonate cages at $22-24^{\circ} \mathrm{C}, 30-40 \%$ humidity, and a 12 -hr light/dark cycle. Standard food and sufficient water were given to all animals.

Rats were randomly divided into four groups, each containing four rats. While the first group was accepted as the control group, with no intervention made and no treatment given, the other three groups received a single dose of intraperitoneal injection of 50 $\mathrm{mg} / \mathrm{kg}$ streptozotocin (Sigma-Aldrich, USA) prepared in citrated solution (pH: 4.5). On the seventh day after the injection, blood sugars were measured with a glucometer (Accu-Chek, Roche) from the 1/3 distal tail of the rats. Rats were considered diabetic upon measurements of serum glucose concentration at $270 \mathrm{mg} / \mathrm{dl}(15 \mathrm{mmol} / \mathrm{l})$ and above. Twelve rats with induced diabetes were further divided into three subgroups. No treatment was given to one of the diabetes-induced groups (group 2). Insulin glargine (Lantus, Sanofi Aventis) as a single subcutaneous injection of $10 \mathrm{U} / \mathrm{kg} /$ day (group 3 ) and Rosiglitazone (Avandia, GlaxoSmithKline) at a dose of $20 \mathrm{mcg} / \mathrm{gr} / \mathrm{day}$ by gavage (group 4) were given to the remaining groups.

$B G$ of the animals were measured twice a day, once in the morning and once in the evening. At the end of the study, the BG measurements of each group were averaged, and this was stated as the last BG. Weight follow-up was performed once a week. Animals were euthanized at the end of the sixth week by taking intracardiac blood under Xylasine hydrochloride $5 \mathrm{mg} / \mathrm{kg}$ anesthesia. The right femurs of the rats were dissected and placed in a solution containing formaldehyde and delivered to the laboratory where the investigation would be performed.

After the bone materials were kept in a $10 \%$ neutral formalin for $72 \mathrm{hr}$, they were transferred to the Decastro Solution and kept in it for 30-45 days by refreshing the solution every day, at the end of this period the materials were allowed to be decalcified. Decalcified bone tissues were embedded in paraffin by transmitting light microscope examination. Sections of $6-7-\mu \mathrm{m}$ thickness were taken from paraffin blocks and stained with hematoxylin-eosin (H\&E) method. Pictures of the obtained samples were taken under Leica DM4000 (Germany) computer-equipped photo-light microscope. Using the Qwin package program of this microscope, bone tissue ratio was calculated in an area of $1 \mathrm{~mm}^{2}$ randomly selected from each glass. In this area, trabecular thicknesses were measured from six randomly selected regions and the average trabecular thicknesses 
were calculated. In addition, femoral neck regions were determined by measuring the farthest distance areas in six areas randomly selected from each glass. Measurements resulted in micron level.

SPSS 11.0 (IBM, Chicago, IL, USA) was used for statistical evaluation of the data. Descriptive data was given as median. Kolmogorov-Smirnov test was applied to evaluate whether the data was parametric. The significance of the relationship between two independent groups was studied using the student's $t$-test and the significance in the data within the group was studied using the paired sample $t$-test. Pearson's correlation test was used to evaluate the relation between study variables. If the $p$-value obtained as a result of the tests was $<0.05$, the test result was considered statistically significant.

\section{Results}

There was no statistically significant difference between baseline BG and body weight among all groups included in the study. In the evaluation carried out at the end of the study, there was no statistically significant difference in BG and body weight compared to the baseline in the control group. In the other groups, there was a significant increase in BG compared to the baseline. There was a statistically significant weight loss in group 2. While there was a statistically significant weight gain in group 3 , there was weight loss in group 4, however, it was not statistically significant (Table 1).

TABLE 1: Blood glucose and body weight among groups at the beginning and end of the study.

\begin{tabular}{|l|c|c|c|c|c|c|} 
& $\begin{array}{c}\text { Beginning } \\
\text { BG (mg/dl) }\end{array}$ & $\begin{array}{c}\text { End BG } \\
\text { (mg/dl) }\end{array}$ & P-value & $\begin{array}{c}\text { Beginning } \\
\text { BW (gr) }\end{array}$ & End BW (gr) & $P$-value \\
\hline Group 1 & 116 & 117 & 0.83 & 259 & 273 & 0.07 \\
\hline Group 2 & 120 & 494 & $\mathbf{0 . 0 0 1}$ & 256 & 209 & $\mathbf{0 . 0 2}$ \\
\hline Group 3 & 108 & 184 & $\mathbf{0 . 0 4}$ & 262 & 275 & $\mathbf{0 . 0 4}$ \\
\hline Group 4 & 111 & 373 & $<0.01$ & 263 & 241 & 0.05 \\
\hline
\end{tabular}

BG: blood glucose, BW: body weight

Group 1 is the control group, group 2 is the diabetic group without any therapy, group 3 is the diabetic group receiving insulin, and group 4 is the diabetic group receiving rosiglitazone.

When the BG levels and body weights of the rats were evaluated at the end of the study, there was a significant increase in the BG values in all three diabetic groups compared to the control group. Blood glucose values of groups 3 and 4 were significantly lower than group $2(p<0.01)$. This decrease was more pronounced in group 3 compared to group 4 and was found to be statistically significant $(p<0.01)$. When the body weights were evaluated at the end of the study, it was found to be significantly lower in groups 2 and 4 compared to the control group. There was no significant difference in body 
weight level of group 3 compared to the control group. Body weights of groups 3 and 4 were significantly higher than group 2 (Table 1).

All groups were compared in terms of trabecular thickness, femoral neck thickness, trabecular area, and percentage of trabecular area (trabecular area/total area) (Table 2). When the control group and group 2 were compared, a statistically significant decrease in the average trabecular thickness was found in group $2(p<0.001)$. When the control group and group 3 were compared, a statistically significant decrease in the average trabecular thickness was found in group $3(p=0.009)$. When the control group and group 4 were compared, a statistically significant decrease in the average trabecular thickness in group 4 was found ( $p<0.001$ ). In addition, it was observed that the trabecular thickness of group 4 was lower than that of groups 2 and 3, but statistically significant difference was observed only between groups 3 and 4 . Although the average femoral neck thickness, trabecular area, and percentage of trabecular area decreased in groups 2,3 , and 4 compared to group 1 , this reduction was not statistically significant.

TABLE 2: Histomorphometric parameters among the groups.

\begin{tabular}{|l|c|c|c|c|} 
& Group 1 & Group 2 & Group 3 & Group 4 \\
\hline $\begin{array}{l}\text { Mean trabecular } \\
\text { thickness }(\mu \mathrm{m})\end{array}$ & 416.8 & $286.7^{a}$ & $327.1^{b}$ & $252.4^{a, c}$ \\
\hline $\begin{array}{l}\text { Mean femoral neck } \\
\text { thickness }(\mu \mathrm{m})\end{array}$ & 1500.2 & 1125.9 & 1456.8 & 1304.7 \\
\hline $\begin{array}{l}\text { Mean trabecular area } \\
\left(\mu \mathrm{m}^{2}\right)\end{array}$ & 560258 & 509070 & 556498 & 469577 \\
\hline $\begin{array}{l}\text { Mean percentage of } \\
\text { trabecular area }(\%)\end{array}$ & 56 & 50.8 & 55.6 & 46.8 \\
\hline
\end{tabular}

${ }^{a} P<0.001$ compared to group $1 ;{ }^{b} P=0.009$ compared to group $1 ;{ }^{c} P=0.02$ compared to group 3

Group 1 is the control group, group 2 is the diabetic group without any therapy, group 3 is the diabetic group receiving insulin, group 4 is the diabetic group receiving rosiglitazone.

Correlation analysis was applied to show the relationship among study variables (Table 3). Accordingly, a significant negative correlation was found between BG and mean trabecular thickness and mean femoral neck thickness values. Although there was a negative correlation between BG and trabecular area, it was not statistically significant. There was no correlation between baseline body weight and histomorphometric data. A significant negative correlation was found between the mean blood glucose level and the final body weight $(p<0.001)$.

Bone trabeculae were observed in their usual structure in the form of irregular wedges in the femoral head sections of the control group. Bone lamellae were prominent in trabeculae (Figure 1A). In greater magnification examinations, cubic shapes, which are the active form of osteoblasts located in one row on the outer surface of the trabecula, 
TABLE 3: Correlation analysis between histomorphometric parameters and blood glucose levels.

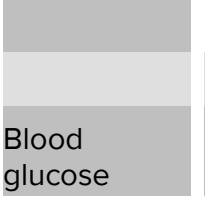

\begin{tabular}{|c|c|}
\hline \multicolumn{2}{|c|}{ Trabecular thickness } \\
\hline$r$ & $P$-value \\
\hline-0.761 & $<0.001$ \\
\hline
\end{tabular}

\begin{tabular}{|c|c|}
\hline \multicolumn{3}{|c|}{ Femoral neck thickness } \\
\hline$r$ & $P$-value \\
\hline-0.562 & 0.024 \\
\hline
\end{tabular}

\begin{tabular}{|c|c|}
\hline \multicolumn{2}{|c|}{ Trabecular area } \\
\hline$r$ & $P$-value \\
\hline-0.382 & 0.14 \\
\hline
\end{tabular}

were observed. Bone lamellae within the trabecula were detected with their normal structure parallel to the long axis of the bone. Among the lamellae, osteocytes located inside their lacunae, and their canalicular structures, were also distinctively normal (Figure 1B). The light microscopic examinations of the untreated diabetes group revealed that the trabeculae were relatively thinned in femoral head sections. In general, bone trabeculae were monitored in their normal order, while missing areas were detected in some places. Basophilic areas in the appearance of primary bone (immature bone) were noted in the trabeculae (Figure 2A). The large-magnification examination revealed that the osteoblasts were squamous, which are their inactive forms. Bone lamellae were observed with their normal structure, while localized osteocytes between the lamellae were determined to be hypertrophic in places (Figure 2B). In light microscopic examinations of the femoral head sections of the diabetic group treated with insulin, the density of the trabeculae was similar to that of the control group, but it was distinguished that they were relatively thin in appearance and showed an irregular course (Figure 3A). In large-magnification examinations, osteoblasts were observed to be active in places with their cubic shapes, and it was determined that osteocytes exhibited a structure similar to the control group. Bone lamellae were normal (Figure 3B). In the light microscopic examinations performed in the diabetic group given rosiglitazone, trabecular loss areas were observed in the trabecular bone tissue of the femoral head, but in general, trabeculae were observed in normal appearance. In some trabecular areas, primary bone tissue areas were observed as basophilic stained (Figure 4A). In large-magnification examinations, osteoblasts were observed in their inactive squamous forms, while bone lamellae were found to be normal. Similar to the normal control group, osteocytes with evident canaliculi were detected in their lacunae (Figure 4B).

\section{Discussion}

Osteoporosis is a frequent metabolic bone disease that progresses with low bone mass and deterioration in bone microarchitecture, causing an increased risk of fracture [16]. It has been known for a long time that type-1 DM reduces BMD in bone and increases the risk of fracture $[1,2]$. In type-2 DM, although there is an increase in BMD in general, 

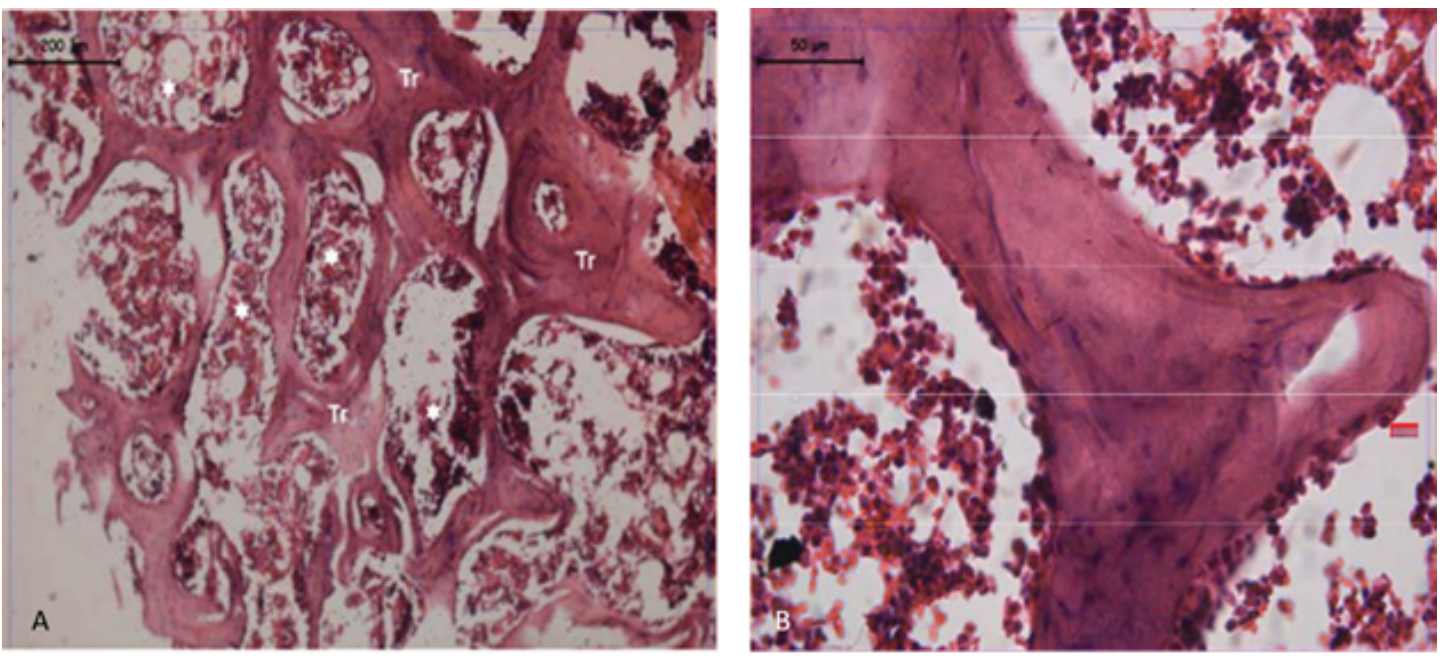

Figure 1: Microscopic view of the femoral neck in the control group. Tr: trabeculae; *Bone marrow tissue (Hematoxylin-Eozin)

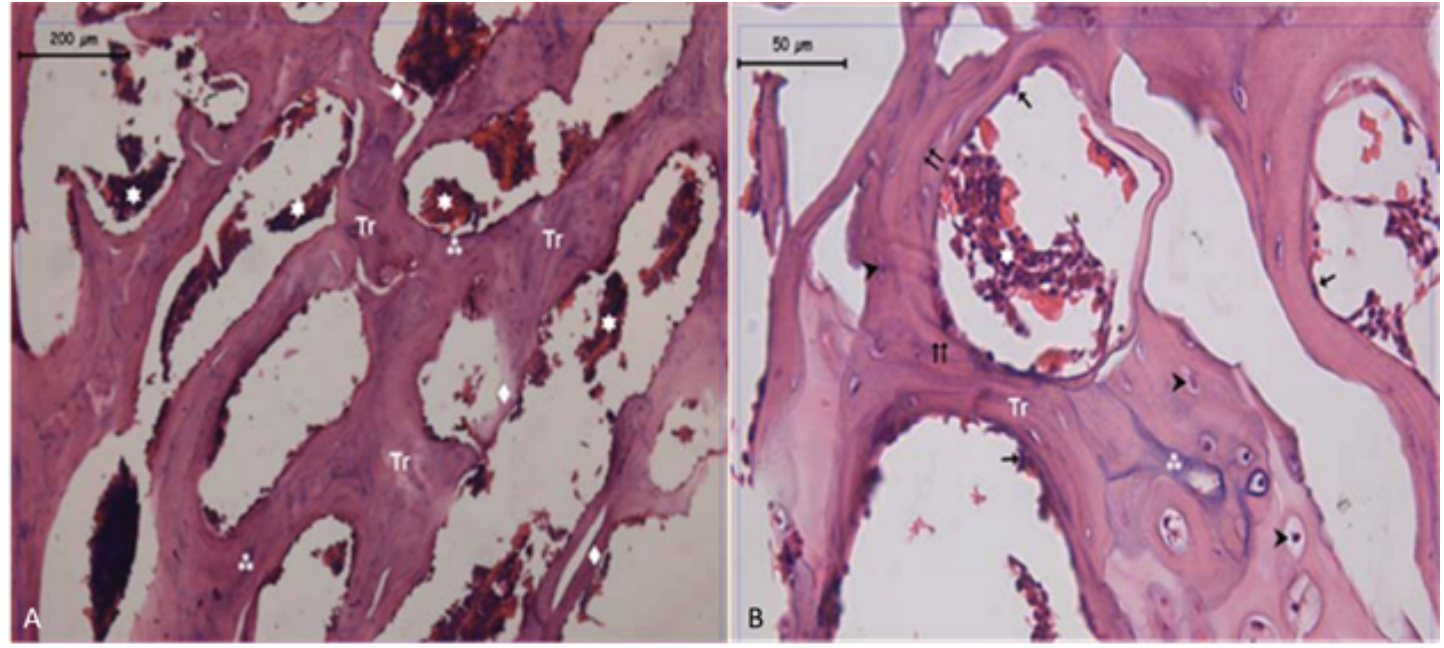

Figure 2: Microscopic view of the femoral neck in the diabetic group without therapy. Tr: trabeculae; *Bone marrow tissue; $\diamond$ Irregular bone trabeculae; Basophilic bone sites. Arrowhead: Osteocyte; Arrow: Osteoblast (Hematoxylin-Eozin).

an increase in the risk of fracture has been detected [3-6, 17, 18]. In previous animal studies, a disruption in histomorphometric parameters in bone was detected both in mice with streptozotocin-induced DM representing type-1 DM and Goto-Kakizaki rats representing type-2 DM $[16,19,20]$.

The mechanisms through how DM causes osteopathy are still being researched. Direct effects of hyperglycemia, insulin insufficiency, low IGF-1 level, and chronic complications of diabetes are among the possible mechanisms that are considered the most $[21,22]$.

In vitro studies have shown that PPARY agonists increase adipogenesis by reducing osteoblastogenesis through the PPARY2 receptors [23]. Increased bone marrow 


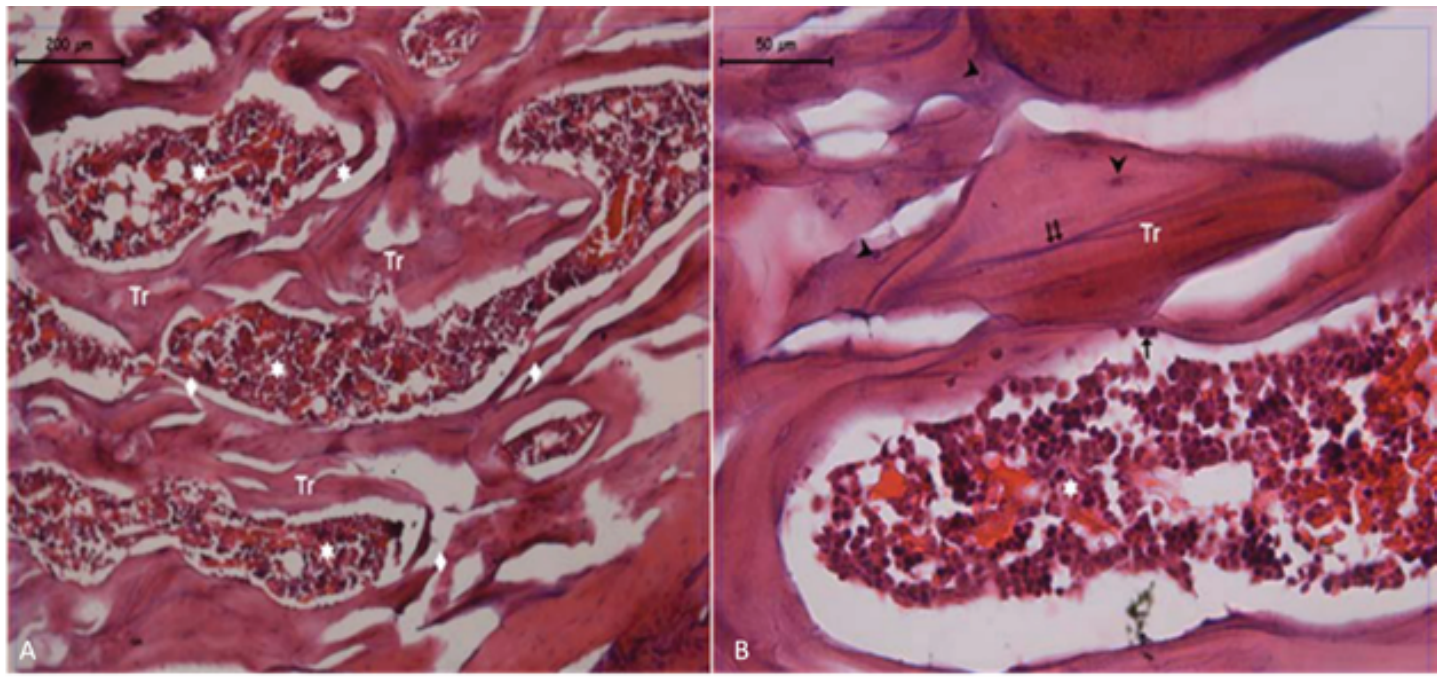

Figure 3: Microscopic view of the femoral neck in the diabetic group treated with insulin. Tr: trabeculae; ${ }^{*}$ Bone marrow tissue; $\diamond$ Irregular bone trabeculae. Arrowhead: Osteocyte; Arrow: Osteoblast (HematoxylinEozin).

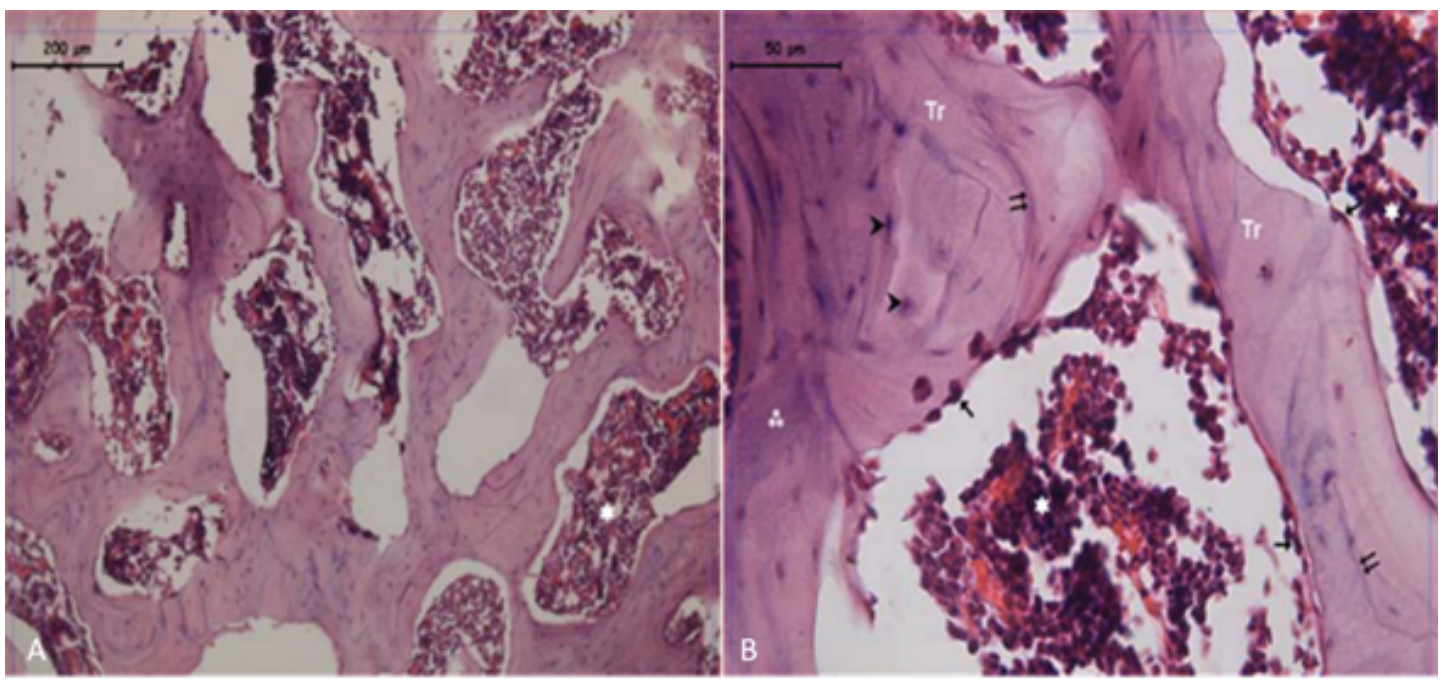

Figure 4: Microscopic view of the femoral neck in the diabetic group exposed to rosiglitazone. Tr: trabeculae; *Bone marrow tissue; $\diamond$ Irregular bone trabeculae; Basophilic bone sites. Arrowhead: Osteocyte; Arrow: Osteoblast (Hematoxylin-Eozin).

adipocyte count with the use of TZDs and decreased bone formation were detected $[12,13,15]$. Negative effects of TZDs on bone are also known by human studies $[24,25]$. TZD has been found to have negative effect on bone in in vitro studies, animal and human experiments.

The effects of TZDs on bone in older mice have been shown to be more pronounced compared to youths and adults [16]. In addition, in one study, rosiglitazone did not have a significant effect on bone in normal rats, whereas adverse effects of the application of rosiglitazone on the bones were found in oophorectomy-performed rats [17]. Also, an increase in bone marrow adipogenesis was detected in the group that underwent 
oophorectomy without medication, and with this finding, it was thought that estrogen deficiency may activate PPARY [17]. It was thought that the administration of rosiglitazone to this group, in which PPAR $\gamma$ activity was increased, had the negative effect on the bone more pronounced. For these reasons, postmenopausal Wistar rats were used in our study to see the effects of TZDs more clearly. In addition, both osteoporosis and DM are more common in the postmenopausal period in humans, and therefore the use of a treatment agent that has a negative effect on bone becomes more important in patients in this group. Therefore, rats in the postmenopausal period, which are at higher risk for osteoporosis, were included in our study. In this study, the effects of diabetes, blood glucose therapy with insulin, and TZD treatment on bone histomorphometric parameters were evaluated using postmenopausal Wistar rats. Since both DM and rosiglitazone have a negative effect on bone, mice were induced diabetes via streptozotocin in order to reveal the effect of rosiglitazone more clearly. To the best of our knowledge, no other study has included postmenopausal streptozotocin induced diabetic Wistar rats to investigate the effect of DM, insulin therapy, and TZD on bone.

The blood glucose levels followed during the study were high in the diabetic group, as expected. In the insulin group, although the blood sugar was significantly lower than the diabetic group, it remained above normal and the blood glucose level after treatment was detected significantly higher than the baseline. Rosiglitazone was used at a dose of $20 \mathrm{mcg} / \mathrm{gr} / \mathrm{day}$, which has previously been shown to provide effective blood glucose reduction in rats [26]. Also in our study, although the TZD group had lower blood glucose values than the untreated diabetes group, it was found that the blood glucose level in this group was significantly higher than the group receiving insulin. No difference was detected between the initial blood glucose level in the control group and the blood glucose levels followed during the study.

At the end of the study, the rats in all groups were compared in terms of trabecular thickness, femoral neck thickness, trabecular area surface, and the ratio of this surface to the whole area. In the diabetic group, all parameters decreased compared to the control group, but only the difference in trabecular thickness was statistically significant. The fact that the data are not significant in other parameters is probably due to the low number of the rats. Also in our study, there is insulin deficiency due to pancreatic damage by applying streptozotocin to rats and it fits more to type-1 DM. The significant reduction in trabecular thickness detected in our study is in line with the BMD-reducing effect of type-1 DM found in the literature [1, 27, 28].

There was an increase in all histomorphometric parameters in the group we were trying to achieve blood glucose regulation with insulin therapy compared to the untreated 
diabetic group. When insulin group was compared to control group, although the trabecular thickness was found to be low, no difference was detected between the trabecular area surfaces. Similarly, decrease in BMD with diabetes and improvement with insulin were determined in a rat study formerly [19].

When the TZD group was evaluated, a decrease was detected in all parameters compared to the control group. While the decrease in trabecular thickness was statistically significant, statistical significance could not be achieved despite the significant decrease in other values. This situation was attributed to the low number of rats in the groups. In the rosiglitazone-administered group, there was a decrease in trabecular thickness and rate of trabecular area compared to the untreated diabetes group, therefore the decrease in this group was interpreted as the effect of TZD with hyperglycemia. In the correlation analysis, a negative correlation was found between the increased blood glucose and trabecular thickness. As a result, it is of note that while the average blood glucose in the TZD group decreased compared to the untreated group, there was disruption in the bone histomorphometric parameters compared to the untreated group. The results are in compliance with the literature [8, 12, 13, 29].

In addition to histomorphometric measurements, the femoral sections of the rats were subjected to histological examination. In this study, bone trabeculae in the control group were observed in their usual structure. Osteoblasts were detected in their active cubic forms. When the untreated diabetes group was examined, areas of thinning of trabeculae and missing areas in trabeculae were found in some places. In addition, immature bone tissues and squamous cells which are the inactive form of osteoblasts were detected in this group. In the insulin-administered group, the density of trabeculae was found similar to the control group, but relative thinning was detected in the trabeculae. In this group, active osteoblasts in similar structure to the control group were detected. When rosiglitazone-administered group was examined, in general, the trabecular structure was in natural appearance although some areas of trabeculae were found to be missing. Immature bone tissue and inactive osteoblasts were observed, similar to the untreated diabetes group. As a result of histological examination, it was found that the presence of diabetes and the use of rosiglitazone caused negative effects on bone continuity by negatively affecting osteoblast and osteocyte activity, and it was observed that an improvement in bone histology can be achieved with insulin. 


\section{Conclusion}

In our study, it was observed that in postmenopausal streptozotocin-induced diabetic Wistar rats, transforming the rats diabetic decreased trabecular thickness and trabecular area rates among bone histomorphometric parameters; improvement of histomorphometric parameters can be achieved by treating diabetic postmenopausal Wistar rats with insulin; histomorphometric parameters worsened with TZD compared to the untreated diabetes group; similar to the histomorphometric findings, in the histological examination, the untreated diabetic group and TZD group had decreased osteoblast activity and the histological findings of the group treated with insulin were similar to the control group. It is known that bone loss with the use of TZD is especially higher in postmenopausal rats, for this reason, special care should be taken before TZD treatment, especially in postmenopausal women, where there is a higher risk of diabetes and osteoporosis.

\section{Acknowledgements}

\section{Ethical considerations}

The study protocol was approved by the Faculty of Medicine Animal Experiments Local Ethics Committee of Gazi University (Code no: G.Ü.ET- 10.055).

\section{Competing interests}

No conflict of interest exists.

\section{Availability of data and material}

The raw data used and/or analyzed during the current study are available from the corresponding author on reasonable request.

\section{Funding}

None. 


\section{Authors' contributions}

DK, BAY, EK, and NÇ worked on the study concept and design or acquisition, analysis, or interpretation of data. DK, GTK, BAY, and NÇ drafted the article and revised it critically for important intellectual content. All authors approved the final version of the article and agree to be accountable for the accuracy and integrity of all aspects of the work

\section{References}

[1] Vestergaard, P. (2007). Discrepancies in bone mineral density and fracture risk in patients with type 1 and type 2 diabetes-a meta-analysis. Osteoporosis International, vol. 18, no. 4 , pp. 427-444.

[2] Miao, J., Brismar, K., Nyrén, O., et al. (2005). Elevated hip fracture risk in type 1 diabetic patients: a population-based cohort study in Sweden. Diabetes Care, vol.28, no.12, pp. 2850-2855.

[3] Grey, A. (2009). Thiazolidinedione-induced skeletal fragility-mechanisms and implications. Diabetes, Obesity \& Metabolism, vol. 11, no. 4, pp. 275-284.

[4] Ahmed, L. A., Joakimsen, R. M., Berntsen, G. K., et al. (2006). Diabetes mellitus and the risk of non-vertebral fractures: the Tromsø study. Osteoporosis International, vol. 17, no. 4, pp. 495-500.

[5] Janghorbani, M., Van Dam, R. M., Willett, W. C., et al. (2007). Systematic review of type 1 and type 2 diabetes mellitus and risk of fracture. American Journal of Epidemiology, vol. 166, no. 5, pp. 495-505.

[6] Nicodemus, K. K., Folsom, A. R., and lowa Women's Health Study. (2001). Type 1 and type 2 diabetes and incident hip fractures in postmenopausal women. Diabetes Care, vol. 24, no. 7, pp. 1192-1197.

[7] Gimble, J. M., Robinson, C. E., Wu, X., et al. (1996). Peroxisome proliferator-activated receptor-gamma activation by thiazolidinediones induces adipogenesis in bone marrow stromal cells. Molecular Pharmacology, vol. 50, no. 5, pp. 1087-1094.

[8] Grey, A. (2008). Skeletal consequences of thiazolidinedione therapy. Osteoporosis International, vol. 19, no. 2, pp. 129-137.

[9] Kawaguchi, H., Akune, T., Yamaguchi, M., et al. (2005). Distinct effects of PPARgamma insufficiency on bone marrow cells, osteoblasts, and osteoclastic cells. Journal of Bone and Mineral Metabolism, vol. 23, no. 4, pp. 275-279. 
[10] Mbalaviele, G., Abu-Amer, Y., Meng, A., et al. (2000). Activation of peroxisome proliferator-activated receptor-gamma pathway inhibits osteoclast differentiation. The Journal of Biological Chemistry, vol. 275, no. 19, pp. 14388-14393.

[11] Okazaki, R., Toriumi, M., Fukumoto, S., et al. (1999). Thiazolidinediones inhibit osteoclast-like cell formation and bone resorption in vitro. Endocrinology, vol. 140, no. 11, pp. 5060-5065.

[12] Ali, A. A., Weinstein, R. S., Stewart, S. A., et al. (2005). Rosiglitazone causes bone loss in mice by suppressing osteoblast differentiation and bone formation. Endocrinology, vol. 146 , no. 3 , pp. 1226-1235.

[13] Lazarenko, O. P., Rzonca, S. O., Hogue, W. R., et al. (2007). Rosiglitazone induces decreases in bone mass and strength that are reminiscent of aged bone. Endocrinology, vol. 148, no. 6, pp. 2669-2680.

[14] Kanda, J., Izumo, N., Kobayashi, Y., et al. (2017). Effect of the antidiabetic agent pioglitazone on bone metabolism in rats. Journal of Pharmacological Sciences, vol. 135, no. 1, pp. 22-28.

[15] Sottile, V., Seuwen, K., and Kneissel, M. (2004). Enhanced marrow adipogenesis and bone resorption in estrogen-deprived rats treated with the PPARgamma agonist BRL49653 (rosiglitazone). Calcified Tissue International, vol. 75, no. 4, pp. 329-337.

[16] Zhang, L., Liu, Y., Wang, D., et al. (2009). Bone biomechanical and histomorphometrical investment in type 2 diabetic Goto-Kakizaki rats. Acta Diabetologica, vol. 46, no. 2, pp. 119-126.

[17] Schwartz, A. V. (2003). Diabetes mellitus: does it affect bone? Calcified Tissue International, vol. 73, no. 6, pp. 515-519.

[18] Epstein, S. and LeRoith, D. (2008). Diabetes and fragility fractures - a burgeoning epidemic? Bone, vol. 43, no. 1, pp. 3-6.

[19] Hamada, Y., Kitazawa, S., Kitazawa, R., et al. (2007). Histomorphometric analysis of diabetic osteopenia in streptozotocin-induced diabetic mice: a possible role of oxidative stress. Bone, vol. 40, no. 5, pp. 1408-1414.

[20] Ying, X., Chen, X., Wang, T., et al. (2020). Possible osteoprotective effects of myricetin in STZ induced diabetic osteoporosis in rats. European Journal of Pharmacology, vol. 866, p. 172805.

[21] Merlotti, D., Gennari, L., Dotta, F., et al. (2010). Mechanisms of impaired bone strength in type 1 and 2 diabetes. Nutrition, Metabolism, and Cardiovascular Diseases, vol. 20, no. 9, pp. 683-690. 
[22] Valcourt, U., Merle, B., Gineyts, E., et al. (2007). Non-enzymatic glycation of bone collagen modifies osteoclastic activity and differentiation. The Journal of Biological Chemistry, vol. 282, no. 8, pp. 5691-5703.

[23] Lecka-Czernik, B., Gubrij, I., Moerman, E. J., et al. (1999). Inhibition of Osf2/Cbfa1 expression and terminal osteoblast differentiation by PPARgamma2. Journal of Cellular Biochemistry, vol. 74, no. 3, pp. 357-371.

[24] Schwartz, A. V., Sellmeyer, D. E., Vittinghoff, E., et al. (2006). Thiazolidinedione use and bone loss in older diabetic adults. The Journal of Clinical Endocrinology and Metabolism, vol. 91, no. 9, pp. 3349-3354.

[25] Eom, Y. S., Gwon, A. R., Kwak, K. M., et al. (2016). Protective effects of vildagliptin against pioglitazone-induced bone loss in type 2 diabetic rats. PloS ONE, vol. 11, no. 12, p. e0168569.

[26] Rzonca, S. O., Suva, L. J., Gaddy, D., et al. (2004). Bone is a target for the antidiabetic compound rosiglitazone. Endocrinology, vol. 145, no. 1, pp. 401-406.

[27] Hofbauer, L. C., Brueck, C. C., Singh, S. K., et al. (2007). Osteoporosis in patients with diabetes mellitus. Journal of Bone and Mineral Research, vol. 22, no. 9, pp. 1317-1328.

[28] Valerio, G., del Puente, A., Esposito-del Puente, A., et al. (2002). The lumbar bone mineral density is affected by long-term poor metabolic control in adolescents with type 1 diabetes mellitus. Hormone Research, vol. 58, no. 6, pp. 266-272.

[29] Kumar, S., Hoffman, S. J., Samadfam, R., et al. (2013). The effect of rosiglitazone on bone mass and fragility is reversible and can be attenuated with alendronate. Journal of Bone and Mineral Research, vol. 28, no. 7, pp. 1653-1665. 